\title{
Automotive Tire Monitoring and Warning System Based on ZigBee Wireless Network
}

\author{
Cheng-shi Luo \\ School of Physics and Electronic Engineering,Taizhou University, Taizhou 318000, China \\ eqyouy@hotmail.com
}

Keywords: TPMS; ZigBee; SP12; MSP430F149; ultra-low power

\begin{abstract}
This paper describes an automotive tire monitoring and warning system based on ZigBee wireless network. By using some components with ultra-low power like ZigBee network device, SP12 Sensor of Infineon which is made for TPMS application, MSP430F149 of TI Company, etc., the problem of inadequate service life of automotive tire pressure detection system is successfully solved. And the keyless door, automatic windows, automatic wipers, etc. of automobile are organized into an intelligent wireless network by applying ZigBee network. This paper describes the main components and provides the hardware diagram.
\end{abstract}

\section{Introduction}

With the development of the times and the progress of society, more and more automobiles have entered the home of ordinary people. According to the statistics from Chinese Ministry of Public Security, over $70 \%$ of the traffic accidents on national highway are caused by tire blowout, and the tire with deficient air is the bane of the tire blowout in the driving. The main function of the intelligent monitoring and warning system of automobile tire pressure is to conduct real-time and automatic monitoring on the pressure and temperature of each tire in the driving, the system can alarm automatically on the condition with hidden danger like a flat tire, low pressure, high pressure, high temperature, etc., which effectively prevents tire blowout and protects the life and property safety of people. And at the same time, this can save fuel, prolong tire life and optimize vehicle performance, thus to provide the added value of automotive safety.

This paper proposes an automotive tire monitoring and warning system based on ZigBee wireless network, the wireless LAN that is combined by the pressure sensor installed inside the tire and various sensors of the automobile integrates the keyless door, automatic windows, automatic wipers, etc. of automobile into a whole. As the address of ZigBee device is unique around the globe, monitoring and warning terminal can identify the tire with abnormal pressure rapidly and at the same time causes no interference among the vehicles.

\section{Zigbee wireless network}

ZigBee technology is the wireless communication technology which is qualified with close distance, low complexity, low power consumption, low data rate and low cost, and it is mainly applied in automatic control. It mainly works in $2.4 \mathrm{GHz}$ ISM frequency range to which no registration is needed, the data rate is $20-250 \mathrm{kbit} / \mathrm{s}$, the maximum transmission range is $10 \sim 75 \mathrm{~m}$ and the typical distance is $30 \mathrm{~m}$. The ZigBee-based wireless module is combined by highly integrated antenna, battery and frequency controller. The WPAN network composed by ZigBee can support up to 254 user nodes, and two-way communication can be achieved by adding a full-function device or a master device. ZigBee mainly reduces frame overhead and carries out strict power management mechanism by reducing the busy-idle ratio of transmitter and the frequency of data transmission, such as reducing the integrated power consumption of the device through shutdown, sleep mode and some other modes.

ZigBee defines two types of physical devices: Full Function Device (FFD), and Reduced Function Device (RFD). Generally speaking, FFD supports any kinds of topological structure, and it can act as a network coordinator that is able to communicate with any devices. RFD is usually only used in star 
network topological structure, it cannot accomplish the function of network coordinator, it can only communicate with FFD, and two RFD cannot communicate with each other. But their internal circuit is less than that of FFD, its internal storage has only little or no energy consumption, which is relatively easier to be achieved and also is more conducive to energy conservation. The most commonly seen topological structures of ZigBee network are star topology and point-to-point topology, and they are shown in Figure 1. For each ZigBee network, at least one FFD network is required to realize network coordination function, and RFD can be used as terminal equipment for reducing system cost.

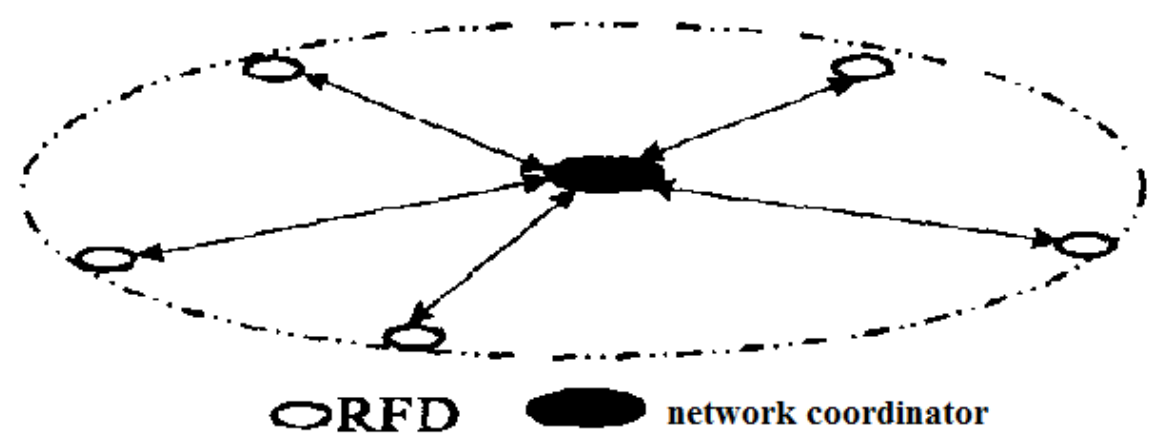

(a)star topology

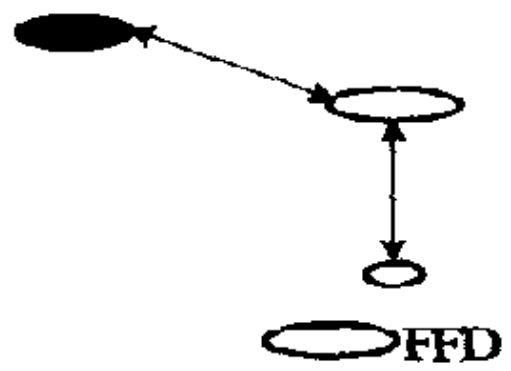

(b)point-to-point topology

Fig 1 Network structure of ZigBee

ZigBee device has its unique advantages in the monitoring system of tire:

(1) low power consumption: the service time of ZigBee device can last as long as six months to two years by only relying on two 5th batteries, which is far more better than other wireless devices. As it is installed inside the tire and the battery cannot be replaced, low power consumption is an essential requirement of tire monitoring.

(2) low cost: the initial cost of ZigBee module is about $\$ 6$, it is estimated that it will drop to $\$ 1.5$ to $\$ 2.5$ soon, and ZigBee protocol is patent-free.

(3) short time delay: the communication delay and the delay from dormant state activation are very short, for typical search equipment, the time delay is $30 \mathrm{~ms}$, the delay from dormant state activation is $15 \mathrm{~ms}$ and the delay from the channel access of active device is $15 \mathrm{~ms}$. Short time delay can give data at the moment of tire blowout, which is conducive to the airbag pop-up and the implementation of some other remedial measures, so as to reduce losses to the minimum. 


\section{Introduction on the tire monitoring system based on ZigBee wireless network}

The whole system is star topological structure. The diagram of hardware is shown in Figure 2.

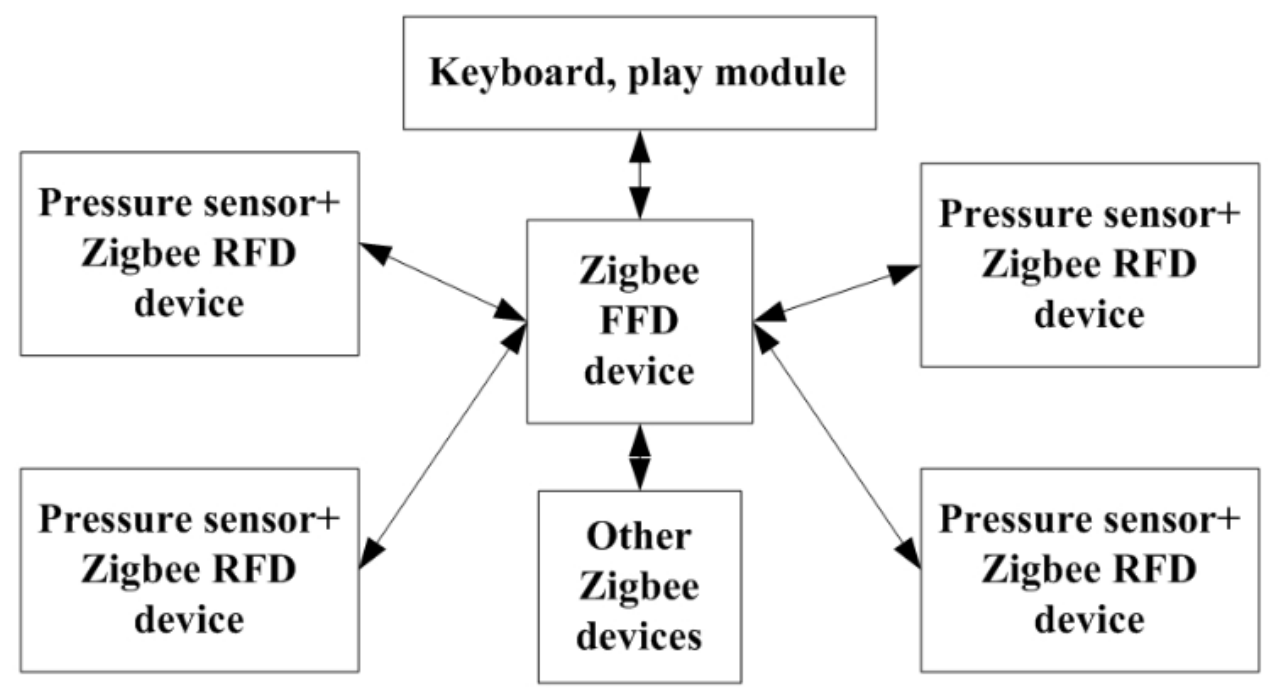

Fig 2 Diagram of the ZigBee wireless intelligent network of automobile

ZigBee FFD is the ZigBee network coordinator of the whole automobile, it is usually installed in the appropriate location of the cab, and there should have good man-machine interface, sound and light alarm and so on. RFD devices includes the pressure monitor terminal installed inside the tire, the electronic lockpick of keyless door, the rain sensor of automatic wiper, the switch of air-condition, temperature sensor, the coordination device of automatic window and so on.

\section{Hardware structure of the tire monitoring terminal based on ZigBee wireless network}

In the tire monitoring system based on ZigBee wireless network, tire pressure sensors and a Zigbee RFD device are installed inside each tire (the position is shown in Figure 3). The entire hardware system is shown in Figure 4:

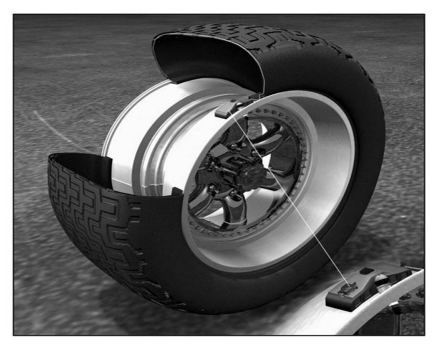

Fig 3 Diagram of the installation position of terminal device

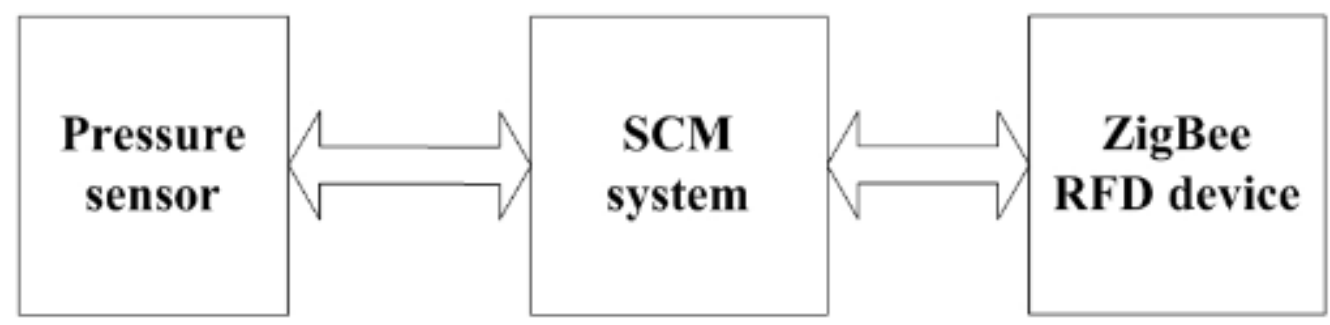

Fig 4 Diagram of the hardware of tire monitoring terminal 
The specific working environment of automotive tires determines the high demand on the pressure sensor of tire pressure real-time monitoring: relatively high accuracy and reliability within wide temperature range and wide power supply and voltage range is required; low power consumption is required; the stability of wireless signal transmission in the harsh environment is required. Therefore, the tire pressure sensor adopts SP12 sensor of Infineon which is made for TPMS application, pressure and acceleration sensor which is silicon micromachined, temperature sensor and battery voltage monitor, so it provides four-in-one sensor function. And a CMOS LSI circuit that can accomplish measurement, signal compensation and adjustment and SPI serial communication interface is provided. Its main parameter indexes are listed as following:

Pressure range: SP12: 100 to $450 \mathrm{Kpa}$

SP12T: 50 to $1400 \mathrm{Kpa}$ (large vehicles)

Acceleration range: $-12 \mathrm{~g}$ to $115 \mathrm{~g}$

Temperature Range: -40 degrees to +125 degrees

Power supply voltage of sensor: $1.8 \mathrm{~V}$ to $3.6 \mathrm{~V}$

Aspect of detection accuracy: silicon piezoresistive pressure sensor is to measure circuit by using the Wheatstone bridge which consists of high-precision semiconductor resistance strain gauge as electromechanical transformation, and its measurement accuracy can achieve $0.01-0.03 \%$ FS.

Aspect of measurement reliability: as the working environment of sensors is harsh, drift will appear in the case of different temperatures, different signal supply voltages, etc., SP12 has a compensation function which can carry on detection and compensation for pressure, acceleration, temperature and supply voltage signal, it accurately provides the correct compensation values for different types of tires in different environments, which effectively ensures the reliability of measurement.

To reduce power consumption, Infineon SP12 Sensor applies wake-up transient operation mode, its power consumption is only 0.6 microamps seconds when it works in sleep operation mode, secondly, acceleration sensor is added to sensor module, the automatic starting of automobile can be realized by using the sensitivity of mass block to movement, and then entering the self-test of system, moreover, if the detected acceleration is very small (it indicates that the automobile has no movement), the system will be made into sleep mode, which greatly reduces the power consumption of system.

SCM system applies the MSP430F149 SCM of TI which is also used in the central processor of system, and as a new type of SCM, MSP430 uses TI Company's latest low power consumption technology. MSP430 works under the voltage of $1.8 \sim 3.6 \mathrm{~V}$ voltage, it has normal operating mode (AM) and four kinds of low power consumption mode (LPM1, LPM2, LPM3, LPM4), when the supply voltage is $3 \mathrm{~V}$, the operating currents of the various modes are respectively: AM: $340 \mathrm{uA}$, LPM1: 70uA, LPM2: 17uA, LPM3: 2uA, LPM4: 0.1uA. The SCM can easily switch between different operating modes. MSP430 has significant advantages in the application of battery-powered and portable device because of its ultra-low power consumption, and it can achieve the real-time access of tire pressure and the protocol stack of ZigBee.

RF device of ZigBee selects CC2420. CC2420 is the first RF transceiver that is accord with the ZigBee standard of $2.4 \mathrm{GHz}$ from ChipconAS Company. The device includes many additional functions, and it is the first RF device that is suitable for ZigBee products. It is based on the SmartRF03 technology of Chipcon Company, and is made by 0.18 um CMOS technology, it needs few external components, and it has stable performance and low power consumption. The selectivity and sensitivity indexes of CC2420 have exceeded the requirement of IEEE802.15.4 standard, which can ensure the validity and reliability of short-distance communication. The wireless communication device that is developed by applying this chip can support up to $250 \mathrm{kbs}$ data transfer rate, thus multi-point fast networking can be achieved. 


\section{Summary}

As the adopted devices in the whole system all have low power consumption and sleep mode, the acceleration sensor of SP12 is used to wake up the whole system. The tire pressure terminal is expected to work for 6-10 years on the condition that its power supply is 750 MA lithium battery, thus it has large marketing and application value.

\section{References}

[1]Hu Dake, C Language Design and Development of MSP430 Series SMC [M], Beijing: Beijing University of Aeronautics and Astronautics Press, 2003

[2]Gu Ruihong, Wireless Network Technology Based on ZigBee and Its Application[J], Application of Electronic Technique,2005,6:1-3 